\title{
On re-normalization in the theory of weak turbulence in plasmas
}

\author{
By JAN VACLAVIK \\ Department of Physics, University of Fribourg, Switzerland
}

(Received 17 February 1975)

The paper compares the methods of re-normalization introduced by the DupreeWeinstock theory of turbulence with those in the theories of Kadomtsev, Rudakov and Tsytovich. It is shown to what extent they are equivalent.

\section{Introduction}

The physics of a high-temperature plasma is described by the Vlasov equation, together with Maxwell's equations, so long as particle correlation can be ignored. Assuming the same holds for them, we may construct a theory of turbulent plasmas by obtaining a solution of the nonlinear Vlasov equation. The first successful step in this direction was taken by so-called weak-turbulence theory, which involves solving the Vlasov and Maxwell equations in a perturbation series. (See e.g. Kadomtsev 1965; Sagdeev \& Galeev 1969; Tsytovich 1970; Davidson 1972.) Individual terms are evaluated using the free particle propagator to integrate along a particle orbit, and the "free" plasmon propagator to solve the nonlinear Maxwell equations by iteration. The free particle propagator is the solution of the Vlasov equation neglecting self-consistent fields, while the free plasmon propagator is the solution of the Maxwell equations neglecting mode coupling.

It is well known that weak-turbulence theory suffers from various divergences, which originate in the use of free propagators. Recent advances in the theory of solving nonlinear problems on plasma turbulence have shown that, to eliminate these divergences, one must in some sense renormalize the free propagators. In what has become known as the Dupree-Weinstock theory, a particle propagator is redefined to include perturbing effects of a self-consistent field (Dupree 1966; Weinstock 1969). Applying another method, Rudakov \& Tsytovich (1971) re-normalized the particle propagator. Finally, in the theory of Makhankov \& Tsytovich (1970), a plasmon propagator is redefined to include the effects of 'turbulent collisions'. (But the first to re-normalize both plasmon and particle propagators was Kadomtsev (1965), in his 'weak-coupling' equations, which he derived from semi-intuitive considerations.)

The work presented here began in an effort to understand the methods introduced by the theories of the above authors, and their inter-relation. The purpose of this communication is to derive the main results of the theories from a unified viewpoint, and to show how far the theories are equivalent. 


\section{Basic equations}

Let us consider a collisionless plasma, in which the distribution function of a species of charge obeys the Vlasov-Poisson equations (for simplicity we neglect the magnetic field):

$$
\begin{gathered}
\left(\frac{\partial}{\partial t}+\mathbf{v} \cdot \frac{\partial}{\partial \mathbf{r}}+\frac{q}{m} \mathbf{E}(\mathbf{r}, t) \cdot \frac{\partial}{\partial \mathbf{v}}\right) f(\mathbf{r}, \mathbf{v}, t)=0, \\
\operatorname{div} \mathbf{E}(\mathbf{r}, t)=4 \pi q\left(\int f(\mathbf{r}, \mathbf{v}, t) d \mathbf{v}-n_{0}\right) .
\end{gathered}
$$

$f(\mathbf{r}, \mathbf{v}, t)$ is the distribution function of species of charge at position $\mathbf{r}$ and velocity $\mathbf{v}$ at time $t . q$ and $m$, respectively, denote the charge (including sign) and mass of the species. $n_{0}$ is the density of a uniform background of neutralizing charge. For notational convenience, we define the linear operator

$$
L(t)=\mathbf{v} \cdot \frac{\partial}{\partial \mathbf{r}}+\frac{q}{m} \mathbf{E}(\mathbf{r}, t) \cdot \frac{\partial}{\partial \mathbf{v}} .
$$

Sometimes we simply denote $f(\mathbf{r}, \mathbf{v}, t)$ by $f(t)$, so that (1) can be written

$$
(\partial / \partial t+L(t)) f(t)=0
$$

Our object is to obtain a set of equations for an ensemble average of $f(t)$, which we denote by $\bar{f}(t)$. Following Weinstock, we introduce a linear operator $A$, which has the property of taking the ensemble average of everything on which it operates. We thus define

$$
A F(t) \equiv\langle F(t)\rangle \equiv \bar{F}(t), \quad A F\left(t_{1}\right) F\left(t_{2}\right) \equiv\left\langle F\left(t_{1}\right) F\left(t_{2}\right)\right\rangle,
$$

where $F(t)$ is an arbitrary function of $t$. The distribution function $f(t)$ can thus be divided into an average part $\bar{f}(t) \equiv A f(t)$ and fluctuating part $f(t)=(1-A) f(t)$ :

$$
f(t)=\vec{f}(t)+f^{\prime}(t) .
$$

The Vlasov equation can then be written as two equations, by operating on both sides of (4) with $A$ and (1-A), to obtain

$$
\begin{gathered}
(\partial / \partial t+\bar{L}(t)) \bar{f}(t)=-A L^{\prime}(t) f^{\prime}(t) \\
(\partial / \partial t+\bar{L}(t)) f^{\prime}(t)=-L^{\prime}(t) \bar{f}(t)+(A-1) L^{\prime}(t) f^{\prime}(t),
\end{gathered}
$$

where

$$
\bar{L}(t) \equiv A L(t), \quad L^{\prime}(t) \equiv(1-A) L(t) .
$$

In what follows, we confine ourselves to the case $\langle\mathbf{E}(\mathbf{r}, t)\rangle=0$. Consequently, $\mathbf{E}^{\prime}(\mathbf{r}, t)=\mathbf{E}(\mathbf{r}, t)$, and the quantities $\bar{L}$ and $L^{\prime}$ are given by the relations

$$
\bar{L}=\mathbf{v} \cdot \frac{\partial}{\partial \mathbf{r}}, \quad L^{\prime}(t)=\frac{q}{m} \mathbf{E}(\mathbf{r}, t) \cdot \frac{\partial}{\partial \mathbf{v}} .
$$

The Poisson equation (2) now reads

$$
\operatorname{div} \mathbf{E}(\mathbf{r}, t)=4 \pi q \int f^{\prime}(\mathbf{r}, \mathbf{v}, t) d \mathbf{v}
$$

Equations (7), (8) and (11), together with (9) and (10), form a complete set of basic equations for the quantities $\bar{f}, f^{\prime}$ and $\mathbf{E}$. We now solve them by means of two different methods. 


\section{Dupree-Weinstock method}

As a first step, we wish to solve (8) for $f^{\prime}(t)$ in terms of $\bar{f}(t)$. Following Birmingham \& Bornatici (1971) we introduce the 'Vlasov' propagator $U\left(t, t_{0}\right)$, which is defined by

and we rewrite (8) as

$$
(\partial / \partial t+L(t)) U\left(t, t_{0}\right)=0, \quad U\left(t_{0}, t_{0}\right)=1,
$$

$$
(\partial / \partial t+L(t)) f^{\prime}(t)=A L^{\prime}(t) f^{\prime}(t)-L^{\prime}(t) \vec{f}(t)
$$

The formal solution of this equation can then be written

$$
f^{\prime}(t)=U\left(t, t_{0}\right) f^{\prime}\left(t_{0}\right)+\int_{t_{0}}^{t} d t_{1} U\left(t, t_{1}\right)\left[A L^{\prime}\left(t_{1}\right) f^{\prime}\left(t_{1}\right)-L^{\prime}\left(t_{1}\right) \bar{f}\left(t_{1}\right)\right] .
$$

Next we neglect the term in (14) associated with the initial value $f^{\prime}\left(t_{0}\right)$, and define the operator $\hat{U}\left(t, t_{0}\right)$ by

$$
\hat{U}\left(t, t_{0}\right) \psi(t)=\int_{t_{0}}^{t} U\left(t, t_{1}\right) \psi\left(t_{1}\right) d t_{1}
$$

$\psi(t)$ is an arbitrary function. Thus, in operator form, (14) reads

$$
f^{\prime}=\hat{U}\left(A L^{\prime} f^{\prime}-L^{\prime} \bar{f}\right) \text {. }
$$

It is straightforward to solve this equation by iteration to any desired order in $L^{\prime}$. With accuracy up to $O\left(L^{\prime 3}\right)$, we obtain

$$
f^{\prime}=-\hat{U}\left(1+A L^{\prime} \hat{U}+A L^{\prime} \hat{U} A L^{\prime} \hat{U}\right) L^{\prime} \bar{f}
$$

We would now like to express the operator $U$ by means of the ensemble-average Vlasov propagator $\bar{U}$. An equation relating $U$ and $\bar{U}$ is obtained by averaging (12), and subtracting the resulting equation from (12) itself:

$$
(\partial / \partial t+L(t))\left(U\left(t, t_{0}\right)-\bar{U}\left(t, t_{0}\right)\right)=\left\langle L^{\prime}(t) U\left(t, t_{0}\right)\right\rangle-L^{\prime}(t) \bar{U}\left(t, t_{0}\right)
$$

Equation (18) formally integrates to

$$
U\left(t, t_{0}\right)=\bar{U}\left(t, t_{0}\right)+\int_{t_{0}}^{t} U\left(t, t_{1}\right)\left[\left\langle L^{\prime}\left(t_{1}\right) U\left(t_{1}, t_{0}\right)\right\rangle-L^{\prime}\left(t_{1}\right) \bar{U}\left(t_{1}, t_{0}\right)\right] d t_{1},
$$

or briefly, in operator form,

$$
U=\bar{U}+\hat{U}\left(\left\langle L^{\prime} U\right\rangle-L^{\prime} U\right)
$$

Equation (20), solved by iteration to accuracy $O\left(L^{\prime 2}\right)$, yields

$$
U=\bar{U}-\hat{\bar{U}} L^{\prime} \bar{U}+\hat{\bar{U}}\left[L^{\prime} \hat{\bar{U}} L^{\prime} \bar{U}-\left\langle L^{\prime} \hat{\bar{U}} L^{\prime} \bar{U}\right\rangle\right]
$$

Upon substituting this $U$ into (17), and neglecting terms of order higher than $L^{\prime 3}$, we obtain

$$
f^{\prime}=-\hat{\bar{U}}\left[1-(1-A) L^{\prime} \hat{\bar{U}}+(1-A) L^{\prime} \hat{\bar{U}}(1-A) L^{\prime} \hat{\bar{U}}-\left\langle L^{\prime} \hat{\bar{U}} L^{\prime} \hat{\bar{U}}\right\rangle\right] L^{\prime} \bar{f}
$$


or, in a more explicit form,

$$
\begin{aligned}
f^{\prime}(t)= & -\int_{t_{0}}^{t} \bar{U}\left(t, t_{1}\right) L^{\prime}\left(t_{1}\right) \bar{f}\left(t_{1}\right) d t_{1}+\int_{t_{0}}^{t} d t_{2} \int_{t_{0}}^{t_{1}} d t_{1} \bar{U}\left(t, t_{2}\right)(1-A) L^{\prime}\left(t_{2}\right) \\
& \times \bar{U}\left(t_{2}, t_{1}\right) L^{\prime}\left(t_{1}\right) \bar{f}\left(t_{1}\right)+\int_{t_{0}}^{t} d t_{3} \int_{t_{0}}^{t_{3}} d t_{2} \int_{t_{0}}^{t_{2}} d t_{1} \bar{U}\left(t, t_{3}\right) \\
& \times\left[\left\langle L^{\prime}\left(t_{3}\right) \bar{U}\left(t_{3}, t_{2}\right) L^{\prime}\left(t_{2}\right) \bar{U}\left(t_{2}, t_{1}\right)\right\rangle-(1-A) L^{\prime}\left(t_{3}\right) \bar{U}\left(t_{3}, t_{2}\right)\right. \\
& \left.\times(1-A) L^{\prime}\left(t_{2}\right) \bar{U}\left(t_{2}, t_{1}\right)\right] L^{\prime}\left(t_{1}\right) \bar{f}\left(t_{1}\right) .
\end{aligned}
$$

After reversing the order of integrations, (23) can be rewritten

$$
\begin{aligned}
f^{\prime}(t)= & -\int_{t_{0}}^{t} d t_{1}\left[\bar{U}\left(t, t_{1}\right)-\int_{t_{1}}^{t} d t_{2} \bar{U}\left(t, t_{2}\right)(1-A) L^{\prime}\left(t_{2}\right) \bar{U}\left(t_{2}, t_{1}\right)+\int_{t_{1}}^{t} d t_{2} \int_{t_{3}}^{t} d t_{3} \bar{U}\left(t, t_{3}\right)\right. \\
& \times\left\{(1-A) L^{\prime}\left(t_{3}\right) \bar{U}\left(t_{3}, t_{2}\right)(1-A) L^{\prime}\left(t_{2}\right) \bar{U}\left(t_{2}, t_{1}\right)\right. \\
& \left.\left.-\left\langle L^{\prime}\left(t_{3}\right) \bar{U}\left(t_{3}, t_{2}\right) L^{\prime}\left(t_{2}\right) \bar{U}\left(t_{2}, t_{1}\right)\right\rangle\right\}\right] L^{\prime}\left(t_{1}\right) \bar{f}\left(t_{1}\right) .
\end{aligned}
$$

Here, the expression in the square bracket represents the expansion of Weinstock's operator $U_{A}$ to second order in $L^{\prime}$ in terms of $\bar{U}$. If we now keep only the lowest-order term in this expansion, and substitute the corresponding $f^{\prime}(t)$ into (7) and (11), we obtain the set of the equations of the 're-normalized' quasilinear approximation:

$$
\begin{gathered}
(\partial / \partial t+\bar{L}) \bar{f}(t)=\int_{t_{0}}^{t} d t_{1}\left\langle L^{\prime}(t) \bar{U}\left(t, t_{1}\right) L^{\prime}\left(t_{1}\right)\right\rangle \bar{f}\left(t_{1}\right) \\
\operatorname{div} \mathbf{E}(\mathbf{r}, t)=-4 \pi q \int d \mathbf{v} \int_{t_{0}}^{t} d t_{1} \bar{U}\left(t, t_{1}\right) L^{\prime}\left(t_{1}\right) \bar{f}\left(t_{1}\right) .
\end{gathered}
$$

The obvious difference between (25) and (26) and the equations of the conventional quasi-linear approximation is that, in the latter, the 'free' particle propagator

replaces $\bar{U}$.

$$
U_{0}\left(t, t_{1}\right)=\exp \left\{\left(t_{1}-t\right) \mathbf{v} \cdot \partial / \partial \mathbf{r}\right\}
$$

Up to this point, the operator $\bar{U}$ is completely unknown. Bearing in mind, however, that the Vlasov propagator satisfies

$$
f(t)=U\left(t, t_{0}\right) f\left(t_{0}\right)
$$

and neglecting the same term as was neglected (14), we can make the approximation

$$
\bar{f}(t)=\bar{U}\left(t, t_{0}\right) \bar{f}\left(t_{0}\right)
$$

Equation (25) can then be expressed in terms of $\bar{U}$, instead of $\bar{f}$, by substituting (29), to obtain

$$
(\partial / \partial t+\bar{L}) \bar{U}\left(t, t_{0}\right)=\int_{t_{0}}^{t} d t_{1}\left\langle L^{\prime}(t) \bar{U}\left(t, t_{1}\right) L^{\prime}\left(t_{1}\right)\right\rangle \bar{U}\left(t_{1}, t_{0}\right) .
$$

Equations (26), (29) and (30) form a complete set for $\bar{U}, \bar{f}$ and $\mathbf{E}$.

In the Dupree-Weinstock theory, (30) is not solved. Instead, one makes use 
of the relationship between $\bar{U}$ and the characteristic trajectories of the Vlasov equation. Let $\mathbf{r}^{*}$ and $\mathbf{v}^{*}$ be the solutions of the characteristic equations

$$
\frac{d \mathbf{r}^{*}}{d \tau}=\mathbf{v}^{*}, \frac{d \mathbf{v}^{*}}{d \tau}=\frac{q}{m} \mathbf{E}\left(\mathbf{r}^{*}, \tau\right) \equiv \mathbf{F}\left(\mathbf{r}^{*}, \tau\right),
$$

with boundary conditions $\mathrm{r}^{*}(\tau=t)=\mathbf{r}, \mathbf{v}^{*}(\tau=t)=\mathrm{v}$. The formal solution of (12) is then

$$
U\left(t, t_{0}\right)=\exp \left\{\left(\mathbf{r}^{*}\left(t_{0}\right)-\mathbf{r}\right) \cdot \frac{\partial}{\partial \mathbf{r}}+\left(\mathbf{v}^{*}\left(t_{0}\right)-\mathbf{v}\right) \cdot \frac{\partial}{\partial \mathbf{v}}\right\}
$$

For brevity we introduce $\pi$, the six-component phase-space vector. Thus,

where

$$
\begin{gathered}
\bar{U}\left(t, t_{0}\right)=\left\langle\exp \left\{\left(\pi^{*}\left(t_{0}\right)-\pi\right) \cdot \frac{\partial}{\partial \pi}\right\}\right\rangle \cong \exp \left\{\left\langle\Delta \pi^{*}\left(t_{0}\right)\right\rangle \cdot \frac{\partial}{\partial \pi}\right. \\
\left.+\frac{1}{2}\left[\left\langle\Delta \pi^{*}\left(t_{0}\right) \Delta \pi^{*}\left(t_{0}\right)\right\rangle-\left\langle\Delta \pi^{*}\left(t_{0}\right)\right\rangle\left\langle\Delta \pi^{*}\left(t_{0}\right)\right\rangle\right]: \frac{\partial}{\partial \pi} \frac{\partial}{\partial \pi}\right\}, \\
\Delta \pi^{*}\left(t_{0}\right)=\pi^{*}\left(t_{0}\right)-\pi
\end{gathered}
$$

In the last form of (33), we have made a cumulant expansion, and dropped cumulants higher than second order. By integrating the characteristic equations (31), $\Delta \pi^{*}\left(t_{0}\right)$ can be expressed in terms of fluctuating fields along a particle trajectory. The operator $\bar{U}$ is then represented in terms of statistical correlations of the fluctuating field.

To proceed further, we make an additional simplification. We assume that $\bar{f}$ changes sufficiently slowly with $\mathbf{r}, \mathbf{v}$ and $t$ that, in (25) and (26), $\bar{f}$ may be removed from both the $\bar{U}$ operation and also from the time integration. With this approximation, (25) and (26) read

where

$$
\begin{gathered}
\left(\frac{\partial}{\partial t}+\mathbf{v} \cdot \frac{\partial}{\partial \mathbf{r}}\right) \bar{f}(\mathbf{r}, \mathbf{v}, t)=\frac{\partial}{\partial \mathbf{v}} \cdot \mathbf{D}\left(\mathbf{r}, \mathbf{v}, t, t_{0}\right) \cdot \frac{\partial \bar{f}(\mathbf{r}, \mathbf{v}, t)}{\partial \mathbf{v}}, \\
\operatorname{div} \mathbf{E}(\mathbf{r}, t)=-\frac{4 \pi q^{2}}{m} \int d \mathbf{v} \frac{\partial \bar{f}(\mathbf{r}, \mathbf{v}, t)}{\partial \mathbf{v}} \cdot \int_{t_{0}}^{t} d t_{1} \bar{U}\left(t, t_{1}\right) \mathbf{E}\left(\mathbf{r}, t_{1}\right),
\end{gathered}
$$

$$
\mathbf{D}\left(\mathbf{r}, \mathbf{v}, t, t_{0}\right)=\int_{0}^{t-t_{0}} d t_{1}\left\langle\mathbf{F}(\mathbf{r}, t) \bar{U}\left(t, t-t_{1}\right) \mathbf{F}\left(\mathbf{r}, t-t_{1}\right)\right\rangle .
$$

Consequently, it is sufficient to consider $\bar{U}$ in the form

$$
\bar{U}\left(t, t_{0}\right)=\exp \left\{\left\langle\Delta \mathbf{r}^{*}\left(t_{0}\right)\right\rangle \cdot \frac{\partial}{\partial \mathbf{r}}+\frac{1}{2}\left[\left\langle\Delta \mathbf{r}^{*}\left(t_{0}\right) \Delta \mathbf{r}^{*}\left(t_{0}\right)\right\rangle-\left\langle\Delta \mathbf{r}^{*}\left(t_{0}\right)\right\rangle\left\langle\Delta \mathbf{r}^{*}\left(t_{0}\right)\right\rangle\right]: \frac{\partial}{\partial \mathbf{r}} \frac{\partial}{\partial \mathbf{r}}\right\}
$$

Let us now formally integrate (31). We have

$$
\Delta \mathbf{r}^{*}(\tau)-\mathbf{v}(\tau-t)=\int_{t}^{\tau}\left(\tau-\tau^{\prime}\right) \mathbf{F}\left(\mathbf{r}^{*}\left(\tau^{\prime}\right), \tau^{\prime}\right) d \tau^{\prime} .
$$

Iterating once, and neglecting the terms of order higher than $F^{2},(38)$ become s

$$
\begin{array}{r}
\Delta \mathbf{r}^{*}(\tau)-\mathbf{v}(\tau-t)=\int_{t}^{\tau} d \tau^{\prime}\left(\tau-\tau^{\prime}\right)\left\{\mathbf{F}\left(\mathbf{r}+\mathbf{v}\left(\tau^{\prime}-t\right), \tau^{\prime}\right)+\frac{\partial \mathbf{F}\left(\mathbf{r}+\mathbf{v}\left(\tau^{\prime}-t\right), \tau^{\prime}\right)}{\partial\left(\mathbf{r}+\mathbf{v}\left(\tau^{\prime}-t\right)\right)}\right. \\
\left.\cdot \int_{t}^{\tau^{\prime}}\left(\tau^{\prime}-\tau^{\prime \prime}\right) \mathbf{F}\left(\mathbf{r}+\mathbf{v}\left(\tau^{\prime \prime}-t\right), \tau^{\prime \prime}\right) d \tau^{\prime \prime}\right\} .
\end{array}
$$


It follows that

$$
\begin{aligned}
\left\langle\Delta \mathbf{r}^{*}(\tau)\right\rangle-\mathbf{v}(\tau-t)= & \int_{t}^{\tau} d \tau^{\prime}\left(\tau-\tau^{\prime}\right) \int_{t}^{\tau^{\prime}} d \tau^{\prime \prime}\left(\tau^{\prime}-\tau^{\prime \prime}\right) \\
& \times\left\langle\mathbf{F}\left(\mathbf{r}+\mathbf{v}\left(\tau^{\prime \prime}-t\right), \tau^{\prime \prime}\right), \frac{\partial \mathbf{F}\left(\mathbf{r}+\mathbf{v}\left(\tau^{\prime}-t\right), \tau^{\prime}\right)}{\partial\left(\mathbf{r}+\mathbf{v}\left(\tau^{\prime}-t\right)\right)}\right\rangle .
\end{aligned}
$$

If we now assume that the turbulence is homogeneous in space and stationary in time, the correlation $\left\langle\mathbf{F}\left(\mathbf{r}_{1}, t_{1}\right) \mathbf{F}\left(\mathbf{r}_{2}, t_{2}\right)\right\rangle$ becomes a function of $\mathbf{r}_{2}-\mathbf{r}_{1}$ and $t_{2}-t_{1}$ only. The relation (40) can then be transformed in such a way that

$$
\begin{array}{r}
\left\langle\Delta \mathbf{r}^{*}(\tau)\right\rangle-\mathbf{v}(\tau-t)=\int_{t}^{\tau} d \tau^{\prime}\left(\tau-\tau^{\prime}\right) \int_{t}^{\tau^{\prime}} d \tau^{\prime \prime} \frac{\partial}{\partial \mathbf{v}}\left\langle\mathbf{F}\left(\mathbf{r}+\mathbf{v}\left(\tau^{\prime \prime}-t\right), \tau^{\prime \prime}\right)\right. \\
\left.. \mathbf{F}\left(\mathbf{r}+\mathbf{v}\left(\tau^{\prime}-t\right), \tau^{\prime}\right)\right\rangle
\end{array}
$$

Performing one of the integrations, we finally obtain

$$
\begin{aligned}
\left\langle\Delta \mathbf{r}^{*}(\tau)\right\rangle-\mathbf{v}(\tau-t) & =\frac{1}{2} \frac{\partial}{\partial \mathbf{v}} \int_{0}^{\tau-t}(\tau-t-z)^{2}\langle\mathbf{F}(\mathbf{r}, t) \cdot \mathbf{F}(\mathbf{r}-z \mathbf{v}, t-z)\rangle d z \\
& \cong \frac{1}{2} \frac{\partial}{\partial \mathbf{v}} \int_{0}^{\tau-t}(\tau-t-z)^{2}\left\langle\mathbf{F}(\mathbf{r}, t) \cdot \mathbf{F}\left(\mathbf{r}^{*}(t-z), t-z\right)\right\rangle d z \\
& \cong \frac{1}{2}(\tau-t)^{2} \frac{\partial}{\partial \mathbf{v}} \int_{0}^{\tau-t}\left\langle\mathbf{F}(\mathbf{r}, t) \cdot \mathbf{F}\left(\mathbf{r}^{*}(t-z), t-z\right)\right\rangle d z
\end{aligned}
$$

The latter approximation follows, because the correlation peaks at $z=0$.

Since $\bar{U} \cong U$, to lowest order in $F$, the diffusion tensor $\mathbf{D}$ (equation (36)) can be written

$$
\begin{aligned}
\mathbf{D}\left(\mathbf{r}, \mathbf{v}, t, t_{0}\right) & \cong \int_{0}^{t-t_{0}} d t_{1}\left\langle\mathbf{F}(\mathbf{r}, t) U\left(t, t-t_{1}\right) \mathbf{F}\left(\mathbf{r}, t-t_{1}\right)\right\rangle \\
& =\int_{0}^{t-t_{0}} d t_{1}\left\langle\mathbf{F}(\mathbf{r}, t) \mathbf{F}\left(\mathbf{r}^{*}\left(t-t_{1}\right), t-t_{1}\right)\right\rangle .
\end{aligned}
$$

By combining (42) and (43), we conclude that

$$
\left\langle\Delta \mathbf{r}^{*}(t-\tau)\right\rangle=-\mathbf{v} \tau-\frac{1}{2} \tau^{2} \partial / \partial \mathbf{v} \operatorname{Tr}\{\mathbf{D}(\mathbf{v}, \tau)\}
$$

for homogeneous and stationary turbulence. $\operatorname{Tr}\{\mathbf{D}\}$ is the trace of $\mathbf{D}$.

Applying the same technique, one can show that

$$
\left\langle\Delta \mathbf{r}^{*}(t-\tau) \Delta \mathbf{r}^{*}(t-\tau)\right\rangle-\left\langle\Delta \mathbf{r}^{*}(t-\tau)\right\rangle\left\langle\Delta \mathbf{r}^{*}(t-\tau)\right\rangle=\frac{2}{3} \tau^{3} \mathbf{D}(\mathbf{v}, \tau) .
$$

Together with (44) and (37), (45) yields

$$
\bar{U}(t, t-\tau)=\exp \left\{-\left(\mathbf{v} \tau+\frac{1}{2} \frac{\partial}{\partial \mathbf{v}} \operatorname{Tr}\{\mathbf{D}(\mathbf{v}, \tau)\} \tau^{2}\right) \cdot \frac{\partial}{\partial \mathbf{r}}+\frac{1}{3} \mathbf{D}(\mathbf{v}, \tau) \tau^{3}: \frac{\partial}{\partial \mathbf{r}} \frac{\partial}{\partial \mathbf{r}}\right\} .
$$

We plug (46) into (36), and expand the $F$ in a Fourier series, to obtain

$$
\begin{aligned}
\mathbf{D}(\mathbf{v}, \tau)= & \int_{0}^{\tau} d \tau^{\prime} \sum_{\mathbf{k}} \hat{k} \hat{k}\left\langle\left|\mathbf{F}_{\mathbf{k}}\right|^{2}\right\rangle \exp \left\{i \tau^{\prime}\left(\omega_{\mathbf{k}}-\mathbf{k} \cdot \mathbf{v}\right)\right\} \\
& \times \exp \left\{-\frac{1}{2} i \tau^{\prime 2} \mathbf{k} \cdot \frac{\partial}{\partial \mathbf{v}} \operatorname{Tr}\left\{\mathbf{D}\left(\mathbf{v}, \tau^{\prime}\right)\right\}-\frac{1}{3} \tau^{\prime 3} \mathbf{k k}: \mathbf{D}\left(\mathbf{v}, \tau^{\prime}\right)\right\} .
\end{aligned}
$$

$\left\langle\left|\mathbf{F}_{\mathbf{k}}\right|^{2}\right\rangle$ is the average square amplitude of the Fourier mode with wave vector $\mathbf{k}$; $\hat{k}$ is the unit vector along $\mathbf{k}$; and $\omega_{\mathbf{k}}$ is the real eigenfrequency. Equation (47) was 
derived by Birmingham \& Bornatici (1972). Dupree (1966) and Weinstock (1969) found the asymptotic expression for $\mathbf{D}$ only. Their formula reads

$$
\mathbf{D}(\mathbf{v}, \infty)=\int_{0}^{\infty} d \tau \sum_{\mathbf{k}} \hat{k} \hat{k}\left\langle\left|\mathbf{F}_{\mathbf{k}}\right|^{2}\right\rangle \exp \left\{i \tau\left(\omega_{\mathbf{k}}-\mathbf{k} \cdot \mathbf{v}\right)-\frac{1}{3} \tau^{3} \mathbf{k} \mathbf{k}: \mathbf{D}(\mathbf{v}, \infty)\right\}
$$

Let us calculate the asymptotic D from (47). Since, in the integral of (47), $\mathbf{D}(\mathbf{v}, \tau)$ occurs in terms that drop off abruptly with $\tau \rightarrow \infty$ we may use the small $\tau$ expansion of $\mathbf{D}(v, \tau)$ in these terms. From (47), we have, in the small $\tau$ limit,

$$
\mathbf{D}(\mathbf{v}, \tau) \cong \sum_{\mathbf{k}} \hat{k} \hat{k}\left\langle\left|\mathbf{F}_{\mathbf{k}}\right|^{2}\right\rangle\left[\tau-\frac{1}{6}\left(\omega_{\mathbf{k}}-\mathbf{k} \cdot \mathbf{v}\right)^{2} \tau^{3}\right]
$$

Substituting (49) into the integral of (47), and letting $\tau \rightarrow \infty$, we obtain

$$
\begin{aligned}
\mathbf{D}(\mathbf{v}, \infty)= & \int_{0}^{\infty} d \tau \sum_{\mathbf{k}} \hat{k} \hat{k}\left\langle\left|\mathbf{F}_{\mathbf{k}}\right|^{2}\right\rangle \exp \left\{i \tau\left(\omega_{\mathbf{k}}-\mathbf{k} \cdot \mathbf{v}\right)\right\} \\
& \times \exp \left\{-i \frac{1}{6} \tau^{5} \mathbf{k} \cdot \sum_{\mathbf{k}^{\prime}} \mathbf{k}^{\prime}\left\langle\left|\mathbf{F}_{\mathbf{k}^{\prime}}\right|^{2}\right\rangle\left(\omega_{\mathbf{k}}{ }^{\prime}-\mathbf{k}^{\prime} \cdot \mathbf{v}\right)\right\} \\
& \times \exp \left\{-\frac{1}{3} \tau^{4} \mathbf{k} \mathbf{k}: \sum_{\mathbf{k}^{\prime}} \hat{k}^{\prime} \hat{k}^{\prime}\left\langle\left|\mathbf{F}_{\mathbf{k}}{ }^{\prime}\right|^{2}\right\rangle\right\}
\end{aligned}
$$

Having found the explicit expression for $\bar{U}(46)$, we can, as a last step, derive a dispersion equation. Substituting (46) into the space and time Fourier transform of (35) taken in the limit $t_{0} \rightarrow-\infty$, we find

$$
\begin{aligned}
\epsilon(\mathbf{k}, \omega) \equiv & 1+\frac{4 \pi q^{2}}{i k^{2} m} \int d \mathbf{v} \mathbf{k} \cdot \frac{\partial \bar{f}}{\partial \mathbf{v}} \int_{0}^{\infty} d \tau \exp \{i \tau(\omega-\mathbf{k} \cdot \mathbf{v})\} \\
& \times \exp \left\{-\frac{1}{2} i \tau^{2} \mathbf{k} \cdot \frac{\partial}{\partial \mathbf{v}} \operatorname{Tr}\{\mathbf{D}(\mathbf{v}, \tau)\}-\frac{1}{3} \tau^{3} \mathbf{k} \mathbf{k}: \mathbf{D}(\mathbf{v}, \tau)\right\}=0
\end{aligned}
$$

$\mathbf{D}(\mathbf{v}, \tau)$ is given by the approximate formula (49). Equations (51) and (34), together with (50), are the main result of this theory.

\section{The method of Kadomtsev, Rudakov and Tsytovich}

\subsection{Re-normalization of the particle propagator}

Let us add an effective turbulent collision frequency operator $\hat{v}$ to both sides of (8):

$$
(\partial / \partial t+\bar{L}+\hat{v}) f^{\prime}=-L^{\prime} \bar{f}+\hat{v} f^{\prime}+(A-1) L^{\prime} \bar{f}^{\prime} .
$$

We also define a propagator $G\left(t, t_{0}\right)$ by

$$
(\partial / \partial t+\bar{L}+\hat{\nu}) G\left(t, t_{0}\right)=0, \quad G\left(t_{0}, t_{0}\right)=1 .
$$

Neglecting the term associated with $f^{\prime}\left(t_{0}\right)$, and further defining an operator $\hat{G}$ in the same manner as $\hat{U},(15)$, the solution to (52) can be written in operator form as

$$
f^{\prime}=\hat{G}\left[-L^{\prime} \bar{f}+\hat{\nu} f^{\prime}+(A-1) L f^{\prime}\right]
$$

Suppose we iterate once on the last term of (54). Then we obtain

$$
f^{\prime}=\hat{G}\left[-L^{\prime} \bar{f}+\hat{\nu} f^{\prime}+(A-1) L^{\prime} \hat{G}\left\{-L^{\prime} \bar{f}+\hat{\nu} f^{\prime}+(A-1) L^{\prime} f^{\prime}\right\}\right] .
$$

If we now identify

$$
\hat{v} f^{\prime}=-\left\langle L^{\prime} \hat{G} L^{\prime}\right\rangle f^{\prime},
$$


(55) becomes

$$
f^{\prime}=\hat{G}\left[-L^{\prime} \bar{f}-\left\langle L^{\prime} \hat{G} L^{\prime}\right\rangle f^{\prime}+(A-1) L^{\prime} \hat{G}\left\{-L^{\prime} \bar{f}-\left\langle L^{\prime} \hat{G} L^{\prime}\right\rangle f^{\prime}+(A-1) L^{\prime} f^{\prime}\right\}\right]
$$

We iterate on this equation once again, and keep only terms up to $O\left(L^{\prime 3}\right)$. We have

$$
f^{\prime}=-\hat{G}\left\{1-(1-A) L^{\prime} \hat{G}+(1-A) L^{\prime} \hat{G}(1-A) L^{\prime} \hat{G}-\left\langle L^{\prime} \hat{G} L^{\prime}\right\rangle \hat{G}\right\} L^{\prime} \bar{f} .
$$

Substituting (56) into (53), we see that the propagator $G$ satisfies

$$
\left(\partial / \partial t+\bar{L}-\left\langle L^{\prime} \hat{G} L^{\prime}\right\rangle\right) G=0 .
$$

From (59), we conclude that $G$ has no stochastic part. The last term in the brackets of (58) can then written $\left\langle L^{\prime} \hat{G} L^{\prime} \hat{G}\right\rangle$. Thus, (58) reads

$$
f^{\prime}=-\hat{G}\left\{1-(1-A) L^{\prime} \hat{G}+(1-A) L^{\prime} \hat{G}(1-A) L^{\prime} \hat{G}-\left\langle L^{\prime} \hat{G} L^{\prime} \hat{G}\right\rangle\right\} L^{\prime} \bar{f} .
$$

If we now rewrite (59) in the more explicit form

$$
(\partial / \partial t+\bar{L}) G\left(t, t_{0}\right)=\int_{t_{0}}^{t} d t_{1}\left\langle L^{\prime}(t) G\left(t, t_{1}\right) L^{\prime}\left(t_{1}\right)\right\rangle G\left(t_{1}, t_{0}\right)
$$

and compare it with (30), we see immediately that $G$ satisfies the same equation as does the propagator $\bar{U}$. Moreover, the stochastic part of the distribution function $f^{\prime}$ is expressed in terms of $\bar{f}$ and $L^{\prime},(60)$, exactly in the same manner as it is done in (22), if we replace $G$ by $\bar{U}$. Hence, we argue that, up to this point, and in the approximation considered, the present method and that of Dupree-Weinstock are completely equivalent. When seeking an explicit form of $G$ in the present theory, however, one does not invoke those properties it has as the average Vlasov propagator. Instead, one endeavours to solve (61).

Let us define a Green's function $g\left(\mathbf{r}, \mathbf{v}, t ; \mathbf{r}_{0}, \mathbf{v}_{0}, t_{0}\right)$ by

$$
\left.\begin{array}{c}
G\left(t, t_{0}\right) \psi\left(\mathbf{r}, \mathbf{v}, t_{0}\right)=\int g\left(\mathbf{r}, \mathbf{v}, t ; \mathbf{r}_{0}, \mathbf{v}_{0}, t_{0}\right) \psi\left(\mathbf{r}_{0}, \mathbf{v}_{0}, t_{0}\right) d \mathbf{r}_{0} d \mathbf{v}_{0}, \\
g\left(\mathbf{r}, \mathbf{v}, t_{0} ; \mathbf{r}_{0}, \mathbf{v}_{0}, t_{0}\right)=\delta\left(\mathbf{r}-\mathbf{r}_{0}\right) \delta\left(\mathbf{v}-\mathbf{v}_{0}\right) .
\end{array}\right\}
$$

$\psi$ is an arbitrary function. Equation (61) is then transformed into

$$
\begin{aligned}
\left(\frac{\partial}{\partial t}+\mathbf{v} \cdot \frac{\partial}{\partial \mathbf{r}}\right) g\left(\mathbf{r}, \mathbf{v}, t ; \mathbf{r}_{0}, \mathbf{v}_{0}, t_{0}\right)= & \left(\frac{q}{m}\right)^{2} \frac{\partial}{\partial \mathbf{v}} \cdot \int_{t_{0}}^{t} d t^{\prime} \int d \mathbf{r}^{\prime} d \mathbf{v}^{\prime} g\left(\mathbf{r}, \mathbf{v}, t ; \mathbf{r}^{\prime}, \mathbf{v}^{\prime}, t^{\prime}\right) \\
& \times\left\langle\mathbf{E}(\mathbf{r}, t) \mathbf{E}\left(\mathbf{r}^{\prime}, t^{\prime}\right)\right\rangle \cdot \frac{\partial}{\partial \mathbf{v}^{\prime}} g\left(\mathbf{r}^{\prime}, \mathbf{v}^{\prime}, t^{\prime} ; \mathbf{r}_{0}, \mathbf{v}_{0}, t_{0}\right) .
\end{aligned}
$$

For homogeneous and stationary turbulence, we conclude, from (63), that

$$
g\left(\mathbf{r}, \mathbf{v}, t ; \mathbf{r}_{0}, \mathbf{v}_{0}, t_{0}\right) \equiv g\left(\mathbf{r}-\mathbf{r}_{0}, t-t_{0}, \mathbf{v}, \mathbf{v}_{0}\right) .
$$

It is then easy to perform the Fourier-Laplace transformation of (63) in space and time. We obtain

$$
\begin{array}{r}
i(\omega-\mathbf{k} . \mathbf{v}) g\left(k, \mathbf{v}, \mathbf{v}_{0}\right)+(2 \pi)^{-4}\left(\frac{q}{m}\right)^{2} \frac{\partial}{\partial \mathbf{v}} \cdot \int d k^{\prime} \mathbf{k}^{\prime} \mathbf{k}^{\prime} I\left(k^{\prime}\right) \int d \mathbf{v}^{\prime} g\left(k-k^{\prime}, \mathbf{v}, \mathbf{v}^{\prime}\right) \\
\cdot \frac{\partial}{\partial \mathbf{v}^{\prime}} g\left(k, \mathbf{v}^{\prime}, \mathbf{v}_{0}\right)=-\delta\left(\mathbf{v}-\mathbf{v}_{0}\right)
\end{array}
$$

where

$$
k=\{\mathbf{k}, \omega\}, \quad d k=d \mathbf{k} d \omega, \quad I(k)=\left\langle|\phi(k)|^{2}\right\rangle .
$$

We have introduced the electric field potential by $\mathbf{E}(k)=-i \mathbf{k} \phi(k)$. 
Equation (64) was solved approximately by Rudakov \& Tsytovich (1971) in the neighbourhood of the resonance $\omega=\mathbf{k}$. v. They showed that

$$
g\left(k, \mathbf{v}, \mathbf{v}_{0}\right) \cong g_{0}(k, \mathbf{v}) \delta\left(\mathbf{v}-\mathbf{v}_{0}\right),
$$

where

$$
g_{0}(k, \mathbf{v}) \cong \int_{0}^{\infty} \exp \left\{i(\omega-\mathbf{k} \cdot \mathbf{v}) \tau-D(\mathbf{k}, \mathbf{v}) \frac{1}{3} \tau^{3}\right\} d \tau
$$

and

$$
\begin{aligned}
D(\mathbf{k}, \mathbf{v})=(2 \pi)^{-4}(q / m)^{2} \int d k^{\prime}\left(\mathbf{k} \cdot \mathbf{k}^{\prime}\right)^{2} I\left(k^{\prime}\right) \int_{0}^{\infty} d \tau \exp \left\{-i\left(\omega^{\prime}-\mathbf{k}^{\prime} \cdot \mathbf{v}\right) \tau\right\} \\
\\
\times \exp \left\{-\frac{1}{3} \tau^{3} D\left(\mathbf{k}-\mathbf{k}^{\prime}, \mathbf{v}\right)\right\} .
\end{aligned}
$$

In lowest order in $D,(67)$ yields

$$
D(\mathbf{k}, \mathbf{v})=(2 \pi)^{-4}(q / m)^{2} \pi \int d k^{\prime}\left(\mathbf{k} \cdot \mathbf{k}^{\prime}\right)^{2} I\left(k^{\prime}\right) \delta\left(\omega^{\prime}-\mathbf{k}^{\prime} . \mathbf{v}\right) .
$$

To compare these results with (46), let us represent the operator $\bar{U}$ by a Green's function $u$, defined by

$$
\bar{U}\left(t, t_{0}\right) \psi\left(\mathbf{r}, t_{0}\right)=\int u\left(\mathbf{r}-\mathbf{r}_{0}, t-t_{0}, \mathbf{v}\right) \psi\left(\mathbf{r}_{0}, t_{0}\right) d \mathbf{r}_{\mathbf{0}} .
$$

Making use of (46), it is easy to show that the Fourier-Laplace transform of $u$ is given by

$$
\begin{aligned}
u(k, \mathbf{v})=\int_{0}^{\infty} d \tau \exp \left\{i(\omega-\mathbf{k} \cdot \mathbf{v}) \tau-i \frac{1}{2} \tau^{2} \mathbf{k} .\right. & \partial / \partial \mathbf{v} \operatorname{Tr}\{\mathbf{D}(\mathbf{v}, \tau)\}\} \\
& \times \exp \left\{-\frac{1}{3} \tau^{3} \mathbf{k k}: \mathbf{D}(\mathbf{v}, \tau)\right\}
\end{aligned}
$$

We see immediately that $u(k, \mathbf{v})$ differs from $g_{0}(k, \mathbf{v}),(66)$. Moreover, according to the assumption made, the operator $\bar{U}$, as given by (46), does not operate in velocity space. In the present theory, such a property of $G$ was not assumed, but demonstrated within the approximation (65).

To proceed further, we now make the same assumption concerning the behaviour of the function $\bar{f}$ with respect to $\mathbf{r}$ and $t$ as we did in $\S 3$, and take $t_{0} \rightarrow-\infty$. It is then simple to derive an equation for $\bar{f}$. Replacing $\bar{U}$ by $G$ in (25), and making use of $(62),(65),(66)$ and $(68)$, we again arrive at (34). But here the diffusion tensor

$$
\begin{aligned}
\mathbf{D}(\mathbf{v})= & (2 \pi)^{-4}(q / m)^{2} \int \mathbf{k} \mathbf{k} I(k) d k \int_{0}^{\infty} \exp \{i(\omega-\mathbf{k} \cdot \mathbf{v}) \tau\} \\
& \times \exp \left\{-\frac{1}{3} \tau^{3} \pi(2 \pi)^{-4}\left(\frac{q}{m}\right)^{2} \int d k^{\prime}\left(\mathbf{k} \cdot \mathbf{k}^{\prime}\right)^{2} I\left(k^{\prime}\right) \delta\left(\omega^{\prime}-\mathbf{k}^{\prime} \cdot \mathbf{v}\right)\right\} d \tau
\end{aligned}
$$

We discuss the question of an equation for the electric field potential (analogous to $(35)$ or $(51))$ in $\$ 4.2$.

\subsection{Re-normalization of the plasmon propagator}

Let us substitute (60) into (11), and perform the Fourier transform of the resulting equation in space and time. Making use of (65), we obtain

$$
\begin{aligned}
\epsilon(k) \phi(k)= & (2 \pi)^{-4} \int d k_{1} d k_{2} \delta\left(k-k_{1}-k_{2}\right) \epsilon\left(k, k_{1}, k_{2}\right)(1-A) \phi\left(k_{1}\right) \phi\left(k_{2}\right) \\
& +(2 \pi)^{-8} \int d k_{1} d k_{2} d k_{3} \delta\left(k-k_{1}-k_{2}-k_{3}\right) \epsilon\left(k, k_{1}, k_{2}, k_{3}\right) \\
& \times\left\{(1-A) \phi\left(k_{1}\right)(1-A) \phi\left(k_{2}\right)-\left\langle\phi\left(k_{1}\right) \phi\left(k_{2}\right)\right\rangle\right\} \phi\left(k_{3}\right),
\end{aligned}
$$


where

and

$$
\begin{gathered}
\epsilon(k)=1+\frac{4 \pi q^{2}}{i m|\mathbf{k}|^{2}} \int d \mathbf{v} g_{0}(k, \mathbf{v}) \mathbf{k} \cdot \frac{\partial \bar{f}}{\partial \mathbf{v}} \\
\epsilon\left(k, k_{1}, k_{2}\right)=-\frac{2 \pi q^{3}}{m^{2}|\mathbf{k}|^{2}} \int d \mathbf{v} g_{0}(k, \mathbf{v})\left\{\mathbf{k}_{\mathbf{1}} \cdot \frac{\partial}{\partial \mathbf{v}} g_{0}\left(k_{2}, \mathbf{v}\right) \mathbf{k}_{2} \cdot \frac{\partial}{\partial \mathbf{v}}\right. \\
\left.+\mathbf{k}_{\mathbf{2}} \cdot \frac{\partial}{\partial \mathbf{v}} g_{0}\left(k_{1}, \mathbf{v}\right) \mathbf{k}_{1} \cdot \frac{\partial}{\partial \mathbf{v}}\right\} \bar{f}
\end{gathered}
$$

$$
\begin{aligned}
\epsilon\left(k, k_{1}, k_{2}, k_{3}\right)=-\frac{4 \pi q^{4} i}{m^{3}|\mathbf{k}|^{2}} \int d \mathbf{v} g_{0}(k, \mathbf{v}) \mathbf{k}_{1} & \frac{\partial}{\partial \mathbf{v}} g_{0}\left(k-k_{1}, \mathbf{v}\right) \\
& \times \mathbf{k}_{2} \cdot \frac{\partial}{\partial \mathbf{v}} g_{0}\left(k_{3}, \mathbf{v}\right) \mathbf{k}_{3} \cdot \frac{\partial \bar{f}}{\partial \mathbf{v}}
\end{aligned}
$$

Had we retained the lowest-order term in $\phi$ only in (11), we should have arrived at (51) with $u(k, \mathbf{v}),(70)$, replaced by $g_{0}(k, \mathbf{v})$. Such an approximation would correspond to the re-normalized quasi-linear theory described in $\S 3$. The present theory also includes the mode-coupling effects in the three-wave approximation, represented by the right-hand side of (72).

We now multiply (72) by $\phi^{*}\left(k^{\prime}\right)$, integrate over $k^{\prime}$, and take the ensemble average. Also, in the resulting equation, we convert the average value of four $\phi$ into products of two $\phi$, by making use of the random-phase approximation. We thus obtain

$$
\begin{aligned}
\epsilon(k) I(k)= & \frac{I(k)}{(2 \pi)^{4}} \int \epsilon\left(k, k_{1}, k,-k_{1}\right) I\left(k_{1}\right) d k_{1}+(2 \pi)^{-8} \\
& \times \int \epsilon\left(k, k_{1}, k_{2}\right)\left\langle\phi^{*}\left(k^{\prime}\right) \phi\left(k_{1}\right) \phi\left(k_{2}\right)\right\rangle \delta\left(k-k_{1}-k_{2}\right) d k^{\prime} d k_{1} d k_{2} .
\end{aligned}
$$

To reduce (76) further requires evaluation of the triple correlation function $\left\langle\phi^{*}\left(k^{\prime}\right) \phi\left(k_{1}\right) \phi\left(k_{2}\right)\right\rangle$. For this, we add to both sides of (72) the term $\eta(k) \phi(k)$; and we assume that the right-hand side of the resulting equation is small. Iterating on it once, we find

$$
\begin{aligned}
\phi^{(1)}(k)=\tilde{\epsilon}(k)^{-1}\left\{\eta(k) \phi^{(0)}(k)+(2 \pi)^{-4} \int d k_{1} d k_{2} \delta\left(k-k_{1}-k_{2}\right) \epsilon\left(k, k_{1}, k_{2}\right)\right. \\
\\
\left.\times(1-A) \phi^{(0)}\left(k_{1}\right) \phi^{(0)}\left(k_{2}\right)\right\}
\end{aligned}
$$

where

$$
\tilde{\epsilon}(k)=\epsilon(k)+\eta(k) \text {. }
$$

If (77) is used to iterate for each of the three $\phi$ in the triple correlation function, and the random-phase approximation is then applied, (76) can be reduced to

$$
\begin{aligned}
\epsilon(k) I(k)=I(k) & (2 \pi)^{-4} \int\left\{\epsilon\left(k, k_{1}, k,-k_{1}\right)+4 \frac{\epsilon\left(k, k_{1}, k-k_{1}\right) \epsilon\left(k-k_{1},-k_{1}, k\right)}{\tilde{\epsilon}\left(k-k_{1}\right)}\right\} I\left(k_{1}\right) d k_{1} \\
& +\frac{2}{(2 \pi)^{4}} \tilde{\epsilon}(k)^{*-1} \int\left|\epsilon\left(k, k_{1}, k_{2}\right)\right|^{2} I\left(k_{1}\right) I\left(k_{2}\right) \delta\left(k-k_{1}-k_{2}\right) d k_{1} d k_{2}
\end{aligned}
$$

If we now identify

$$
\eta(k)=-(2 \pi)^{-4} \int\left\{\epsilon\left(k, k_{1}, k,-k_{1}\right)+4 \frac{\epsilon\left(k, k_{1}, k-k_{1}\right) \epsilon\left(k-k_{1},-k_{1}, k\right)}{\tilde{\epsilon}\left(k-k_{1}\right)}\right\} I\left(k_{1}\right) d k_{1},
$$


(79) becomes

$$
|\tilde{\epsilon}(k)|^{2} I(k)=\frac{2}{(2 \pi)^{4}} \int\left|\epsilon\left(k, k_{1}, k-k_{1}\right)\right|^{2} I\left(k_{1}\right) I\left(k-k_{1}\right) d k_{1} .
$$

Equations (80) and (81) constitute a complete system for the unknowns $\eta(k)$ and $I(k)$.

If we replace the 're-normalized' plasmon propagator, $\tilde{\epsilon}(k)$, by the 'free' one, $\epsilon(k)$, in (79), we arrive at a single equation for $I(k)$, which was derived by Rudakov \& Tsytovich (1971). Equations of the same type as (80) and (81) were obtained by Kadomtsev (1965) and Makhankov \& Tsytovich (1970), applying a method different from that used here.

This work was supported by the Swiss National Science Foundation.

\section{REFERENCES}

Birmingham, T. J. \& Bornatior, M. 1971 Phys. Fluids, 14, 2239.

Birmingham, T. J. \& Bornatict, M. 1972 Phys. Fluids, 15, 1778.

Davidson, R. C. 1972 Methods in Nonlinear Plasma Theory. Academic.

Dupree, T. H. 1966 Phys. Fluids, 9, 1773.

Kadomtsev, B. B. 1965 Plasma Turbulence. Academic.

Maknankov, V. G. \& Tsytovich, V. N. 1970 Nuclear. Fusion, 10, 405.

Rudakov, L. I. \& Tsytovich, V. N. 1971 Plasma Phys. 13, 213.

SAGDEEv, R. Z. \& GALEev, A. A. 1969 Nonlinear Plasma Theory. Benjamin.

Tsytovich, V. N. 1970 Nonlinear Effects in Plasma. Plenum.

WeINstock, J. 1969 Phys. Fluids, 12, 1045. 